\title{
Más allá de la victimización de niñas y niños en contextos de conflicto armado: potenciales para la construcción de paz*
}

Beyond Victimizing Children in Contexts of Armed Conflict: PeaceBuilding Potencials

Recepción: 01 Marzo 2015 | Aprobación: 03 Mayo 2017

\author{
María Alejandra Fajardo Mayo \\ Universidad de Manizales, Colombia \\ ORCID: http://orcid.org/0000-0003-0323-1085 \\ Mónica Piedad Ramírez Lozano \\ Universidad de Manizales, Colombia \\ María Isabel Valencia Suescún \\ Universidad de Manizales, Colombia \\ María Camila Ospina-Alvarado \\ Universidad de Manizales, Colombia \\ ORCID: http://orcid.org/0000-0002-7271-151X
}

\footnotetext{
a Autor de correspondencia. Correo electrónico: mafajardom@gmail.com
}

Para citar este artículo: Fajardo Mayo, M. A., Ramírez Lozano, M. P., Valencia Suescún, M. I., \& OspinaAlvarado, M. C. (2018). Más allá de la victimización de niñas y niños en contextos de conflicto armado: potenciales para la construcción de paz. Universitas Psychologica, 17(1), 1-14. https://doi.org/10.11144/Ja veriana.upsy17-1.mavn

\section{RESUMEN}

El artículo presenta la revisión de estudios correspondiente a una de las fases del proyecto de investigación en el cual se enmarca, que buscó aproximarse al estado del arte del conocimiento producido en Colombia entre 2002 y 2012 sobre la identidad y subjetividad de niños y niñas en torno a la paz y la democracia, en contextos de conflicto armado. Se presenta la afectación de sus derechos en dichos contextos desde las áreas: existencia, desarrollo, participación y protección. Se aporta una lectura crítica a la mirada tradicional centrada en la vulneración de derechos y el posicionamiento de los niños y niñas como víctimas, invitando a identificar los potenciales individuales y colectivos con las que cuentan ellos, ellas y sus familias.

Palabras clave

niñez; conflicto armado; derechos humanos; construcción de paz.

\begin{abstract}
The article presents the review of studies corresponding to one of the phases of the research project in which it is framed, which sought to approximate the state of the art of knowledge produced in Colombia between 2002 and 2012 on the identity and subjectivity of children around to peace and democracy in contexts of armed conflict. Children and youth rights are affected in these contexts from the areas of existence, development, protection andparticipation. The study provides a critical reading of the traditional view focused on the violation of rights and the positioning of children as victims, inviting to identify the individual and collective potentials with which they and their families are provided.

Keywords

childhood; armed conflict; human rights; peace-building.
\end{abstract}


El presente artículo retoma la revisión de varios estudios, realizada en el marco de la investigación titulada "Procesos de construcción social de la niñez en contextos de conflicto armado en el Eje Cafetero, Antioquia y Área Metropolitana de Bogotá: la paz, la reconciliación y la democracia desde la perspectiva de narrativas generativas de niños y niñas", cuyo objetivo general fue comprender las formas en que la niñez construye y narra sus identidades y subjetividades, en miras a la construcción de paz, democracia y reconciliación en contextos de conflicto armado. Este proyecto hizo parte del programa "Sentidos y prácticas políticas de niños, niñas y jóvenes en contextos de vulnerabilidad en el Eje Cafetero, Antioquia y Bogotá: un camino posible de consolidación de la democracia, la paz y la reconciliación mediante procesos de formación ciudadana", co-financiado por Colciencias y el Consorcio Niños, Niñas y Jóvenes Constructores de Paz: democracia, paz y reconciliación de la Fundación Centro Internacional de Educación y Desarrollo Humano (Cinde), Universidad de Manizales y Universidad Pedagógica Nacional.

Los estudios referidos en el presente artículo son resultado de la primera fase del proyecto de investigación, enfocada a establecer y actualizar el estado del arte del conocimiento producido en Colombia sobre la identidad y subjetividad generativa de niños y niñas, en torno a la paz y la democracia en contextos de conflicto armado. Esta revisión permite comprender las características y el impacto de dicho conflicto, especialmente en los niños y niñas, teniendo como base problemas estructurales como la distribución inequitativa de las riquezas y la exclusión social, los cuales se suman a una precaria o nula presencia del Estado en ciertos territorios, generando condiciones que mantienen y perpetúan la vulneración de los derechos (Niño, 2012). Ha sido desde la década de los 90 que se empiezan a visibilizar en el ámbito académico y social temas como el reclutamiento y la vinculación de niños y niñas al conflicto armado (Montoya, 2008), aspectos que se deben tener en cuenta para comprender las dimensiones del conflicto armado y el impacto del mismo en los niños y niñas.

El artículo expone inicialmente la situación de los niños y las niñas en cuanto a la vulneración directa e indirecta de sus derechos en cuatro áreas. Por un lado, se exponen los derechos de existencia, los cuales contemplan la salud, la alimentación y la sexualidad; los derechos de desarrollo que dan cuenta de la educación, el estado de la escuela y el papel de la familia y las prácticas culturales, en las posibilidades que tienen los niños y niñas para estudiar, teniendo en cuenta el impacto del accionar de los grupos armados tanto legales como ilegales cuando irrumpen en los escenarios de socialización de la niñez; los derechos de participación, escasamente estudiados, pero desde los cuales se establecen diferencias de acuerdo al género, la generación y la participación en espacios públicos o privados y, por último, los derechos de protección, amenazados principalmente, según Niño (2012), por homicidios y afectación de la integridad física, violencia sexual basada en género, desplazamiento forzado y reclutamiento ilegal.

Posteriormente, se plantea la concepción que se tiene de los niños y niñas desde la victimización y su papel pasivo frente a situaciones de emergencia, y las formas como ellos y ellas constituyen sus subjetividades desde contextos de conflicto armado y su simbología. Finalmente, se da un lugar para desplegar la perspectiva del proyecto en relación con lo encontrado en la revisión de los estudios. En este sentido, se presenta un acápite orientado a las potencias de los niños y niñas en contraposición a las concepciones que se tienen de ellos y ellas desde la victimización. También se abarcan las posibilidades que tienen para constituirse como sujetos políticos y cómo se enriquece este proceso desde las potencias con las que también cuentan las familias, las escuelas y las comunidades.

\section{Método}

Dentro de las acciones de la primera fase del proyecto de investigación, se planteó 
un levantamiento de información secundaria que permitiera identificar aquellos estudios relacionados con la niñez y el conflicto armado entre los años 2002 y 2012, teniendo como primer filtro de selección aquellos realizados en el territorio nacional. Se priorizaron los informes de investigación de los organismos nacionales e internacionales, relacionados con la temática del estudio, algunos artículos de revistas indexadas y el principal libro de resultados de investigación desarrollado en Colombia en función de la primera infancia en contextos de conflicto armado. En este sentido, se retomaron 15 documentos, entre los cuales se encuentran informes de investigación provenientes de fuentes institucionales como la Defensoría del Pueblo en asocio con la Unicef, el Observatorio de Territorios Étnicos de la Pontificia Universidad Javeriana, la Agencia de la ONU para los Refugiados en Latinoamérica (ACNUR), la Asociación "Colombia soy yo", la Asociación de Voluntariado y Cooperación internacional NATs per... Onlus, la Coalición contra la vinculación de niños, niñas y jóvenes al conflicto armado en Colombia (Coalico), la Revista Latinoamericana de Ciencias Sociales, Niñez y Juventud en Redalyc, la revista Opinión Jurídica de la Facultad de Derecho de la Universidad de Medellín en Redalyc, la Revista Latinoamericana de Psicología, el Repositorio Institucional de la Universidad del Rosario, WATCHLIST on Children and Armed Conflict, Organización de Estados Iberoamericanos (OEI) y Save the Children.

Se excluyeron artículos que no se enfocaban en la niñez, sino en la juventud; que referían los impactos del conflicto armado en otros países y no en Colombia; que no se habían finalizado en el período propuesto y por ende no contaban con una publicación o con el informe en dicho período o que se desprendían de programas de formación de pregrado y maestría. Aproximadamente, se revisaron 35 documentos que no se tuvieron en cuenta por los criterios mencionados. En este sentido, una limitación es el número de investigaciones revisadas que se incluyó finalmente en el informe y en el presente artículo; limitación que se debió a que gran número de las investigaciones rastreadas no cumplió con los parámetros de inclusión. Un punto fuerte es la rigurosidad del análisis en la revisión bibliográfica desarrollada, como se podrá observar en el siguiente procedimiento.

A continuación, se presentan los pasos seguidos para la revisión bibliográfica como método de investigación científica. En primer lugar, se construyó una matriz de revisión que contó con información descriptiva (referencia bibliográfica según normas APA, título, autores, revista, link o URL) y con las siguientes dimensiones: temas, resumen, palabras clave, metodología, perspectiva teórica, perspectiva epistemológica, objetivo de la investigación, planteamientos centrales, frases y párrafos principales, discusión, conclusiones y otras observaciones. En segundo lugar, se realizó un rastreo de los informes de investigación, artículos y libros que respondían a los criterios definidos para el estado del arte. En tercer lugar, se diligenciaron las matrices para la recopilación de la información correspondiente a cada investigación. En cuarto lugar, se analizó la información consignada en las matrices, se revisaron las categorías de la presente investigación: construcción de paz, democracia y reconciliación; construcción de subjetividades e identidades; conflicto armado y política pública, a la luz de de los estudios priorizados, y surgieron algunas categorías emergentes: contextos, territorios y actores del conflicto armado colombiano; estado de vulneración de los derechos de los niños y niñas; marco normativo relacionado con niñez y conflicto armado; acciones de la sociedad civil; socialización política y constitución de subjetivad; formas de nombrar a los niños y niñas en contextos de conflicto armado y de la victimización a las potencias. En quinto lugar, se escribió un informe del estado del arte que contempló tanto los resultados de las investigaciones en función de las categorías en mención, como las orientaciones epistemológicas, metodológicas y teóricas de los estudios revisados. En sexto lugar, se escribió el presente artículo que busca visibilizar las afectaciones de los niños y niñas en contextos de conflicto armado, a partir de las áreas de derecho, 
y a la vez mostrar sus potencias y posibilidades de transformación.

Por lo anterior, en el presente artículo, se priorizaron las siguientes categorías: estado de vulneración de los derechos de los niños y niñas; socialización política y constitución de subjetividad y de la victimización a las potencias, las cuales se desarrollan a continuación.

\section{Resultados y discusión}

A continuación, se presentan algunos resultados parciales de la investigación, en función de la revisión bibliográfica como método de investigación científica, correspondiente a una de las fases del estudio, específicamente aquella encaminada a la construcción del estado de arte. Dichos resultados se ponen en diálogo con algunas aproximaciones teóricas, a manera de discusión.

Estado de vulneración de los derechos de los niños y las niñas

En relación con el impacto del conflicto armado, diversos estudios se interesan por evaluar el estado de garantía o vulneración de los derechos de los niños y las niñas que viven en entornos de conflicto armado o que son víctimas directas del conflicto, como es el caso de los rescatados de los grupos armados ilegales (GAI) (Defensoría del Pueblo, 2002; Hernández \& Restrepo, 2011; Niño, 2012; Torrado, Camargo, Pineda, \& Bejarano, 2009; WATCHLIST on children and armed conflict, 2004). De acuerdo a las investigaciones revisadas, los derechos de los niños y las niñas colombianas han sido vulnerados en todas sus áreas (existencia, desarrollo, protección y participación).

\section{Los derechos de existencia}

En el área de existencia, se evidencia vulneración del derecho a la salud y a la alimentación. El conflicto genera vulneración en el acceso a la salud, por causas estructurales que se empeoran en las zonas de conflicto. Las investigaciones reportan afectación en la salud mental de niños y niñas que han sufrido desplazamiento forzado o reclutamiento que les dificulta o impide su adaptación a nuevos entornos socioculturales o a la vida civil cuando son desvinculados de los GAI (Niño, 2012; Torrado et al., 2009).

Los derechos sexuales y reproductivos se ven más vulnerados en zonas de conflicto. Se reportan tasas mayores de mortalidad materna, prevalencia de VIH (SIDA), de mujeres gestantes con VIH y de embarazos adolescentes. Durante el reclutamiento en los GAI, las niñas son objeto de uso forzado de medios para prevenir embarazos y abortos (Niño, 2012; WATCHLIST on children and armed conflict, 2004).

Respecto a la alimentación, en zonas de conflicto se encuentra una tasa mayor de desnutrición crónica, asociada a vulnerabilidad demográfica, a fragilidad por baja disponibilidad de alimentos y de acceso a los mismos por restricciones que generan los GAI y por extorsiones que deben pagar para llevar alimentos a sus casas. En las áreas urbanas, este poco acceso se relaciona con los bajos ingresos que tienen las familias (Ila Martínez, Arias, Núñez, \& Caicedo, 2009; Niño, 2012). Las fumigaciones con glifosato afectan la disponibilidad de alimentos en tanto queman los cultivos alimentarios ubicados en las áreas de fumigación (Niño, 2012). Se genera morbilidad, consecuencia de las fumigaciones con glifosato y se reportan nuevas causas de mortalidad debidas a malformaciones genéticas atribuidas a esta contaminación (Niño, 2012; Torrado et al., 2009). El conflicto armado empeora las condiciones estructurales que son causa de la inseguridad alimentaria (Ila et al., 2009).

\section{Los derechos de desarrollo}

Las investigaciones reportan afectación del derecho a la educación en términos de deserción escolar y desescolarización causada por desmotivación por el estudio, extra edad, bajos ingresos familiares y necesidad de trabajar, entre otros (Defensoría del Pueblo, 2006; Niño, 2012). 
El conflicto armado vulnera el derecho a la educación por la militarización o los combates que impiden el desarrollo de las clases o el arribo de niños y niñas a las escuelas, por el uso de estas como campamentos o sitios de reunión de los GAI, por el daño de la infraestructura debido a los combates y la propaganda que realizan estos grupos. En las zonas de conflicto armado, la inestabilidad de los maestros y maestras es alta, lo que dificulta el acceso a la educación (Defensoría del Pueblo, 2002; Niño, 2012). Las acciones armadas realizadas en o en torno a las escuelas, como masacres, convierten estos sitios en símbolos de terror, las señales de la violencia, la sangre, las ruinas, convierten estos lugares en sitios de memoria y dolor (Niño, 2012).

En zonas de alta intensidad del conflicto como Tibú, las grandes distancias que niños, niñas y jóvenes deben recorrer para ir y volver de sus escuelas se convierten en factor de riesgo, en tanto son rutas del narcotráfico y del microtráfico de armas. Son barreras para el acceso a la educación los costos relacionados con uniformes, útiles y dinero para el refrigerio (Niño, 2012).

Igualmente, las transformaciones en la estructura familiar por cuenta del conflicto armado hace que los niños, niñas y jóvenes deban asumir responsabilidades del cuidado del hogar y los hermanos y hermanas menores, así como la realización del trabajo doméstico (Niño, 2012). También cobran importancia los aspectos culturales que le dan más relevancia al aprendizaje de oficios caseros o del campo que a los aprendizajes escolares, lo cual, como lo expresan Torrado et al. (2009), "puede afectar las oportunidades de los niños para entrar en contacto con bienes culturales como la música y la lengua escrita, y con actividades enriquecedoras para su desarrollo integral" (p. 82).

Para Coalico y la Comisión Colombiana de Juristas (CCJ) (2009), las acciones cívicomilitares y educativas que las fuerzas militares colombianas realizan en las escuelas y colegios ponen en riesgo la población escolar. También reportan la injerencia de miembros adscritos a las fuerzas militares en los contenidos educativos, en varios municipios del Chocó y de Bolívar. El secretario general de la ONU reporta la misma situación de riesgo para la vida de niños y niñas por la presencia de fuerzas militares en las instituciones educativas, en los departamentos de Antioquia, Arauca, Córdoba y Norte de Santander (ONU, 2010).

Las escuelas en entornos de conflicto armado se convierten en escenarios para la presencia de los GAI que buscan nuevos adeptos y reclutas a través de la intimidación, el control social y los engaños, como fue constatado en 12 departamentos del país.

La guerrilla ingresa a las escuelas para enganchar niños. Un ejemplo de ello que yo conocí sucedió en la Macarena (Meta) donde el frente séptimo y el frente Yarí de las FARC ocupaban los alrededores cuando los niños salían al descanso, y les comenzaban a decir que se fueran para la guerrilla que allá tendrían todo y que no tendrían que volver a estudiar para tener plata y armas nuevas. Eso es malo porque los separan de la escuela y de la familia. (Coalico \& CCJ, 2009, p. 51)

La Defensoría del Pueblo (2006) al revisar el derecho a la educación de los niños, niñas y jóvenes encontró que este ha sido vulnerado por los propios grupos armados al reclutar a niños, niñas y jóvenes, porque han impedido su asistencia a la escuela, y por lo tanto el período de reclutamiento fue un tiempo muerto en términos de educación formal. El Estado, la familia y la sociedad han vulnerado también este derecho, en tanto las principales causas para la deserción escolar son la pobreza, la falta de oportunidades, la violencia y la negligencia familiar.

La misma fuente considera que la escuela vulnera el derecho a la educación al no cumplir con la aceptabilidad de la educación, por no armonizar el currículo con enfoque de derechos. Al mismo tiempo, reconoce el esfuerzo del Instituto Colombiano de Bienestar Familiar y del Ministerio de Educación por lograr el acceso al sistema educativo de los niños, niñas y jóvenes desvinculados de los grupos armados, a través de la coordinación interinstitucional (Defensoría del Pueblo, 2006).

En relación con los derechos de desarrollo, Torrado et al. (2009) consideran que una de las principales violaciones que el conflicto armado 
produce en los niños y niñas menores de 6 años es al derecho al juego y esparcimiento, escenarios importantes para la socialización y constitución de subjetividad. Este derecho se ve vulnerado principalmente por la plantación de minas antipersona en sitios cercanos a viviendas y escuelas.

\section{Los derechos de participación}

A pesar de ser reconocidos como derechos fundamentales para el desarrollo de los niños, niñas y jóvenes, y como una herramienta de gran potencia para su prevención, protección y atención en el conflicto armado, su vulneración es poco estudiada y reconocida (Niño, 2012). Este investigador reconoce diferencias por género y generación en las posibilidades de participación de niñas y jóvenes en espacios públicos, en tanto culturalmente se considera el espacio privado de mayor seguridad para ellas, en relación con los actores armados.

Ser niño o niña en estos municipios, significa estar amenazado por una violación permanente de sus derechos y ser niña o joven mujer, significa en sí mismo estar limitada para el desarrollo de su potencial como ciudadana en lo público y permanecer en riesgo constante de ser violentada allí o en el ámbito privado. (Niño, 2012, p. 148)

Otros factores que dificultan su participación ciudadana tienen que ver con la asignación de roles relacionados con la reproducción de la mentalidad patriarcal, que busca restringir el contacto de las niñas y mujeres jóvenes con pares del sexo opuesto, y la falta de espacios reales de participación (Niño, 2012).

\section{Los derechos de protección}

Siguiendo a Niño (2012) los derechos de protección de los niños y las niñas en el marco del conflicto armado colombiano han sido vulnerados por cuatro fenómenos: homicidios y afectación de la integridad física, violencia sexual basada en género, desplazamiento forzado y reclutamiento ilegal. Los participantes de la investigación realizada por esta autora reportaron la vulneración de sus derechos a la vida, la libertad y el trabajo, al igual que los sentimientos de temor, miedo y la sensación de inseguridad.

La ONU (2010) reporta para 2010 el asesinato de niños por los grupos llamados por el gobierno colombiano bandas delictivas (Los Rastrojos), que solo en el departamento de Córdoba realizaron 10 matanzas en las cuales murieron 9 niños entre 13 y 17 años. En el mismo año, también fueron asesinados niños en el departamento del Cauca, y estos hechos violentos han significado desplazamientos de mujeres y niños.

El uso de minas antipersona por parte los GAI son otra causa de muerte y daño a los menores. Su uso está relacionado con los cultivos ilícitos, así como por la presencia de infraestructura estratégica para los GAI, como oleoductos y estaciones eléctricas. Los niños y niñas, en particular, son vulnerables a pararse sobre minas no marcadas mientras caminan a la escuela, al jugar en zonas contaminadas o al trabajar en el campo (Coalico \& CCJ, 2008; Niño, 2012; WATCHLIST on children and armed conflict, 2004).

En relación con la violencia sexual, Niño (2012) la visibiliza como una vulneración de derechos que tiene implícita discriminación de género y de generación, siendo las niñas y las jóvenes las más vulnerables dentro de todas las mujeres. Los delitos de este tipo se configuran en una amenaza permanente para las niñas y jóvenes habitantes tanto de la zona urbana como rural.

Coalico y CCJ (2009), a partir de informes de diversas organizaciones nacionales e internacionales que participan en la mesa de "Mujer y conflicto armado", corroboran que la socialización de la fuerza pública con las comunidades afecta de manera especial a mujeres, jóvenes y niñas que son objeto de violencia sexual, discriminación y tratos degradantes. La Defensoría del Pueblo (2006) encontró, en este sentido, que el maltrato (físico, psicológico y sexual) realizado por la familia u otros adultos cuidadores se presenta en mayor proporción en las mujeres que en los hombres 
desvinculados de los grupos armados, lo que da cuenta de una mayor vulnerabilidad de las mujeres sustentada en la inequidad de género que históricamente se mantiene en las familias y que se reproduce en los grupos armados.

La violencia sexual por género es utilizada como táctica militar y/o política. La Corporación Humanas, en su estudio sobre las acciones del Bloque Catatumbo entre 1999 y 2004, identificó que la violencia sexual se comete en el marco de masacres o ataques colectivos, como táctica de tortura para obtener información, como expresión de control sobre el territorio por el establecimiento de lazos de parentesco a partir de embarazos. La prostitución y el tráfico de niñas son fenómenos asociados al conflicto armado y a sus expresiones: desplazamiento forzado y reclutamiento ilícito (Niño, 2012).

La revisión anterior de los derechos afectados a los niños y niñas en contextos de conflicto armado, da cuenta de una lectura desde su posicionamiento como víctimas del conflicto.

\section{Rol pasivo y de víctimas de los niños y las niñas}

Es frecuente encontrar estudios que desde la mirada proteccionista y la perspectiva de derechos posicionan a los niños y niñas como sujetos beneficiarios o víctimas (Defensoría del Pueblo, 2002, 2006; Ila et al., 2009; Montoya, 2008; ONU, 2010; Romero \& Castañeda, 2009; Torrado et al., 2009; WATCHLIST on children and armed conflict, 2004). Si bien esto es requerido para su reconocimiento y para la restitución de sus derechos, en los procesos de implementación de las políticas públicas de protección a la niñez afectada por el conflicto armado, también puede llegar a inmovilizar procesos de gestión de sus propias vidas.

Los niños y niñas son mencionados de varias formas, resaltando sus sufrimientos y los impactos negativos del conflicto sobre sus vidas. Son considerados como sujetos beneficiarios, sujetos de intercambio, jóvenes guerreros o víctimas en condición especial, y no se destaca esa otra parte de sus vidas en donde siguen siendo seres con capacidades y potencialidades para seguir adelante a pesar de las huellas de la guerra.

La condición de víctimas favorece procesos de reinserción social a través de programas especializados de protección que movilicen acciones institucionales como es el caso que se plantea al ser designados como sujetos beneficiarios por voluntad de desvinculación (Defensoría del Pueblo, 2002).

En los estudios revisados, se encuentran diferencias marcadas en lo que es ser víctima del conflicto armado por múltiples condiciones y el uso de los niños, niñas y jóvenes en la guerra. La OIT citada por la Defensoría del Pueblo hace alusión a los niños y las niñas reclutados como sujetos trabajadores (Defensoría del Pueblo, 2002), cuando afirma:

(...) la Organización Internacional del Trabajo - OIT - proclamó el Convenio 182 de 1999 relativo a la obligación de los Estados Parte de emprender en forma inmediata todas las medidas necesarias para erradicar las peores formas de trabajo infantil, dentro de las cuales se incluye la de reclutar y utilizar personas menores de 18 años para que participen en los conflictos armados. (Defensoría del Pueblo, 2002, p. 1)

Otra forma de designar su condición es la que hace alusión al sujeto pasivo del delito (Defensoría del Pueblo, 2002) que lo hace beneficiario de toda clase de soportes y ayudas en la restitución de los derechos y como sujeto necesitado de reinserción social, como resultado de la desvinculación:

(...) el Estado cuenta con instrumentos nacionales e internacionales que deben ser observados y aplicados por las autoridades judiciales y administrativas a las que les compete la protección y restitución de los derechos de los menores de edad que en cualquier condición se desvinculen de los grupos alzados en armas, ya que son sujetos pasivos del delito de reclutamiento ilícito. Por lo tanto, estos niños y niñas demandan del Estado en su conjunto el restablecimiento de los derechos que les fueron conculcados por quienes, de manera forzada o voluntaria, permitieron su vinculación a los grupos armados. (Defensoría del Pueblo, 2002, p.1) 
La Defensoría del Pueblo (2002), citando Código del Menor, en su estudio deja abierta la discusión acerca de la inespecificidad del manejo del niño involucrado en el conflicto armado como niño infractor, por lo cual es tratado como tal en la ley penal o como niño en situación de abandono o peligro, refiriendo que es el juez quien, en su criterio, remite a instituciones de reeducación o a programas para abordar la situación irregular.

Estos modos de nombrar a los niños desde su vulneración de derechos se constituyen en elementos fundantes de sus subjetividades. El presente estudio asume, sin embargo, que los niños y las niñas, a pesar de haber vivido de manera directa o indirecta el contexto de conflicto armado, tienen en sí mismos y en sus relaciones, potencias y recursos que aportan a la construcción de subjetividades alternativas a la violencia y a la victimización.

$\mathrm{Si}$ las comprensiones se limitaran a esta perspectiva, las capacidades y potencialidades de la niñez para buscar caminos apartados de la guerra quedarían segadas por la influencia del conflicto armado sobre su vida. Esto, teniendo en cuenta el lenguaje de victimización y pasividad con el que se hace referencia a los niños y las niñas, siendo escasa la exaltación de sus recursos como agentes relacionales con potencial de transformación de sí mismos, sus familias, sus escuelas y sus comunidades.

\section{Socialización política y constitución de subjetividad}

Las investigaciones revisadas muestran cómo la vinculación de los niños, niñas y jóvenes a los GAI incide en sus procesos de desarrollo y en la configuración de su personalidad. Se visibilizan algunos intereses investigativos identificados en el estado del arte relacionados con la construcción de significados, sentidos y prácticas en torno a la formación de identidades personales y colectivas que permiten comprender los procesos a través de los cuales los niños y niñas en contextos de conflicto armado construyen su subjetividad, y también en las opciones que tienen para participar como sujetos con agencia en la construcción de posibilidades distintas a la violencia (Alvarado et al., 2012).

Es decir, se habla de un proceso continuo e inacabado, que en palabras de Berger y Luckmann (1999) permite que los sujetos internalicen la realidad y cuenten además con la posibilidad de construir y deconstruir los significados que a esta se le asignan de forma colectiva, lo cual, en el contexto de conflicto armado, conlleva al reconocimiento que tienen los niños y niñas para interpretar sus experiencias, tomar decisiones y manifestarlas en sus espacios y actividades cotidianas. Pero se trata de decisiones que no involucran solo al sujeto individual, sino que impactan el tejido de las relaciones humanas y en términos generales, el fin práctico de toda actividad política que es la conducción de una vida justa y buena (Arendt, 2005, 2008).

\section{El lugar de la simbología}

Se encuentran planteamientos relacionados con la idea de que la interacción con las fuerzas armadas en la vida de los niños, niñas y jóvenes incide en la interiorización de valores relacionados con la imagen de soldado valiente que se configuran en imágenes de poder, libertad y autonomía, constituyendo la vida militar como un estilo de vida deseable. El carácter simbólico representado, por ejemplo, en el uso de armas y de uniformes ofrece a los y las adolescentes atributos como el respeto, el reconocimiento, el poder, el sentido de pertenencia y el estatus que los ubica a nivel de los adultos (Defensoría del Pueblo, 2002, 2006).

Algunas de las reflexiones investigativas han centrado su mirada en el lenguaje desde la socialización política de los niños, niñas y jóvenes, para plantearse nuevos interrogantes investigativos que atiendan a los discursos, como posibilidad de identificación de variables influyentes en la guerra y la violencia política:

¿Cómo es el discurso histórico de los jóvenes y las jóvenes guerreros y guerreras? ¿Cómo es el discurso sobre la justicia y la injusticia, sobre el 
Estado y los gobiernos, sobre el contrato social implícito en que crecieron y fueron socializados? (Castellanos \& Torres, 2008, p. 555)

Algunos estudios visibilizan los relatos de los niños, niñas y jóvenes a partir de sus experiencias a fin de interpretar los motivos de vinculación y las múltiples formas de actuar a partir de constitución de subjetividad y socialización política. Es tal el caso de Moreno, Carmona y Tobón (2010) quienes, en su estudio sobre la vinculación de las niñas a los GAI, notan distanciamientos de las razones que dan diversos estudios explicativos al respecto y plantean en su ejercicio interpretativo como las niñas, por ejemplo, refirieron verse interesadas por aspectos estéticos que guiaron sus elecciones. Los relatos se vieron atravesados por su experiencia amorosa de cortejo, la esperanza de tener un núcleo de amigos o compartir nuevas experiencias, movilizando a más miembros de la familia hacia los caminos que elegidos, lo cual es una transformación de la mirada victimizante a una que muestra la posibilidad de acción de las niñas desde sus propios significados de realidad y elecciones.

En este sentido, se considera a los niños y niñas con capacidades diversas de acción, orientadas por sus propias capacidades de sentir, expresar, crear y actuar, en diálogo con lo que sucede en sus relaciones en diferentes contextos como la vida en la escuela, en la familia y en la comunidad. En un mundo de interacciones de mutua influencia, editan y reeditan sus identidades y subjetividades.

\section{De la victimización a las potencias}

Como se ha dicho anteriormente, los niños y niñas en contexto de conflicto armado son nombrados de diversas formas de acuerdo a las perspectivas teóricas utilizadas en las interpretaciones investigativas y esto se convierte en potencial de acción de movilizaciones sociales, impacto en políticas públicas o el planteamiento de nuevos horizontes para la investigación. Pensar en las formas como el lenguaje se inserta en la construcción de realidades (Gergen, 2007) lleva a encontrar en los significados asignados potenciales de movilización en las formas de actuar, simbolizar y sentir de los y las jóvenes en situación de conflicto armado, razón por la cual es necesario abrir un espacio para presentar las formas en que los niños y niñas son nombrados y las transformaciones que se dan en torno a estas. En esta medida, se plantea el lenguaje como eje de construcción de nuevas realidades, considerando importante visibilizar eso otro que con menor frecuencia se nomina y que los y las convierte en sujetos con capacidades y potenciales. Se trasciende así hacia su reconocimiento como sujetos éticos y políticos con posibilidad de creación de nuevos horizontes y futuros.

Algunas transformaciones hacia el niño y la niña como sujetos políticos

Aunque en menor frecuencia se piensa a los niños y niñas como sujetos con potencialidad de participación y toma de decisiones, sí se observan avances investigativos hacia un mayor potencial de acción, lo cual los y las ubicaría como sujetos políticos. Es tal el caso que plantea Niño (2012) quien visibiliza la necesidad de hacer una "apuesta por su actoría social, acciones concretas encaminadas a fortalecer sus capacidades y habilidades para construir caminos de paz y con ello apostar por la prevención del conflicto" ( $\mathrm{p}$. 173). Busca generar un distanciamiento de la perspectiva adultocéntrica, planteando el valor por el reconocimiento de la capacidad de los niños y niñas para pensar en sus posibilidades, a partir de lo que los rodea: "Se da la posibilidad dentro de la investigación de recrearnos de manera compartida con la visión que los niños y en especial las niñas y adolescentes tienen de su contexto" (Niño, 2012, p. 48).

A pesar de que las búsquedas investigativas en su mayoría sujetan los procesos a las miradas victimizantes, o como lo expresara Carli (2003) acerca de la instalación de la representación del niño que en este caso la ejerce el Estado en la medida en que las acciones se pueden ver enmarcadas en la necesidad de proteger, atender, prevenir, educar y fortalecer las diferentes 
acciones y programas, se notan acciones de empoderamiento a partir de iniciativas de participación de los niños y niñas, como por ejemplo:

[d]iseño de un programa, con cobertura nacional, de promoción de sus derechos, con miras a interiorizar en ellos elementos de protección personal que les permita defenderse de estos abusos y que promueva la denuncia ante las autoridades educativas, de policía, administrativas o judiciales. (Defensoría del Pueblo, 2002, p. 40)

Hernández y Restrepo (2011) encuentran desde la resiliencia una vía alterna para el tratamiento e intervención social que puede plantear nuevos retos y percepciones para los profesionales sociales y de la salud. Por su parte, Moreno et al. (2010) asumen una postura interaccionista para abordar las motivaciones de las niñas que se vinculan a los GAI, es decir, se asigna a la elección de la niña un potencial de acción y decisión que se hace en función de sus expectativas y de las significaciones que ellas mismas le dan como punto de partida para actuar o programar sus actos así sean adecuados o equivocados. Esta es una visión que les permite tener la virtud de gestionar sus intenciones y razonar frente a la realidad de sus elecciones.

WATCHLIST on children and armed conflict (2004) destaca el Movimiento de Niños por la Paz, el cual llevó a cabo el 25 de octubre de 1996 unas elecciones especiales conocidas como "El mandato por la paz y los derechos de los niños", en las cuales, a través de sus votos, los niños expresaron que sus tres prioridades eran el derecho a disfrutar de la vida y de una buena salud, el derecho a la paz y a la protección y el derecho al amor y a la familia. Este movimiento ha sido nominado al Premio Nobel de la Paz, pero no ha logrado tener el impacto que desea.

Estas experiencias permiten considerar la capacidad que tienen los niños y niñas de participar políticamente no solo de formas análogas al modelo democrático como votar para elegir o decidir, sino otras acciones relacionadas con el reconocimiento de sí mismos, de las capacidades personales y de tomar decisiones basadas en el impacto que estas pueden tener en sus vidas y en la de otros y otras.

\section{Potencias en las familias}

Son escasos los estudios que visibilizan la capacidad de la familia más allá de ser considerada un núcleo de protección o un objeto más de fortalecimiento estatal. La Defensoría del Pueblo (2002) reconoce el valor de la familia como espacio protector de los niños, niñas y jóvenes y plantea la necesidad de potencializarla de modo que estos y estas puedan ingresar y mantenerse en el sistema escolar y transformando sus patrones de crianza para erradicar el maltrato infantil.

Este estudio establece la necesidad de articular acciones entre escuela y familia para prevenir el reclutamiento, a través de la adecuación de sus proyectos educativos institucionales a las expectativas, posibilidades e intereses de las localidades. Se plantea la necesidad de diseñar un programa de cobertura nacional de promoción de los derechos de los niños $y$ niñas, con el fin de que se interioricen en sus integrantes elementos de protección personal que les permitan defenderse de abusos y de allí puedan capacitarse para la denuncia ante las diferentes autoridades educativas, de policía, administrativas o judiciales (Defensoría del Pueblo, 2002).

Este tipo de iniciativas llevaría a la estimulación de la autodirección del grupo familiar, pues, como lo plantea Londoño (2005) (citado en Alvarado et al., 2012), ver a la familia únicamente como receptora de apoyos hace que se dé una respuesta temporal a las necesidades de sus integrantes y no le permite responsabilizarse ni empoderarse de sus propios procesos de transformación.

Esto conduce a pensar la relación de la niñez, la familia y la escuela a partir de una perspectiva ecológica (Bronfrenbrenner, 1987), desde la cual el desarrollo humano se da empezando por la relación de la persona con el ambiente y considerando formas no lineales ni jerárquicas, sino de mutua influencia y transformación. 
Potencias en la escuela y comunidad

El estudio realizado por el Observatorio de Territorios Étnicos (2010) invita a la reflexión, desde la experiencia de San Onofre, para pensar cómo los maestros expresaron su afecto con la comunidad, por lo cual se desplazaron de sus propias necesidades individuales hacia la asistencia comunitaria, lo cual denota el valor por la solidaridad y el poder de la unidad.

Se expresa el valor del grupo y la conciencia colectiva como potencial de resistencia cuando visibilizan la posibilidad de construir y crear nuevas opciones educativas y para la vida, como se expresa en estas líneas:

A pesar de esto los profesores encontraron formas de resistirse y enviar un mensaje de rebeldía a sus estudiantes, que seguramente fue entendido más allá de la resistencia y comprendió el ámbito de lo ético, de lo social o de lo político. (Observatorio de Territorios Étnicos, 2010, p. 27)

De otra parte, la Defensoría del Pueblo (2002) encuentra la educación como espacio propicio para el empoderamiento, para que niños, niñas y jóvenes puedan informarse de sus derechos y vigilar su cumplimiento, sobre el cuidado de su cuerpo mediante la educación sexual y el uso del tiempo libre a partir de escuchar las opiniones de los niños y niñas.

Coalico y CCJ (2009) encuentran como potencias para la protección de los niños, niñas y jóvenes, las redes de protección constituidas en organizaciones de la sociedad civil, que cuentan con herramientas de valoración de riesgo, análisis de vulnerabilidades y modelos de autoprotección, a partir de los cuales se han identificado amenazas y riesgos relacionados con el uso de la niñez por GAI y estatales. Igualmente, plantea la potencia de la creación y fortalecimiento de redes de protección comunitarias.

Partiendo de lo anterior y dando cabida al lenguaje como construcción de realidad (Gergen, 2007), es posible pensar por las formas de suceder de la vinculación, en la fuerza de los significados de los niños, niñas y jóvenes para asumir su papel de integrase, participar o emanciparse de la misma dinámica cuando se privilegia su oportunidad de desvinculación. Del mismo modo, es posible identificar cómo, a partir de las mismas estructuras y planteamientos de políticas e instrumentos jurídicos, se podrían ver afectadas sus subjetividades desde los posicionamientos que asuman para el ejercicio de participación y ciudadanía y desde los modos de ser nombrados y nombradas.

\section{Conclusiones}

Los estudios revisados abordan el impacto del conflicto armado en los niños y las niñas en términos de vulneración de sus derechos en las áreas de vida, desarrollo y protección. El impacto del conflicto armado sobre los derechos de participación no es un tema abordado en estos estudios.

El conocimiento producido en Colombia, en relación con la vinculación de los niños y las niñas al contexto del conflicto armado, como lo demuestran los estudios anteriormente mencionados, reflejan a la niñez desde la inacción y desde lógicas en las que se los nombra como sujetos pasivos del delito, beneficiarios por voluntad de desvinculación, trabajadores, entre otros, lo cual alienta la participación del Gobierno y las múltiples acciones asistenciales requeridas para el restablecimiento de sus derechos, pero en menor medida resalta la manera en que ellos y ellas logran generar procesos de paz, reconciliación y democracia que rompan con la naturalización de la violencia.

La presente investigación, tras revisar los modos en los que aparecen posicionados los niños y niñas en contexto de conflicto armado en diversos estudios, hace un llamado a las implicaciones que tiene el estudiarlos y estudiarlas únicamente desde la vulneración de sus derechos. Esta lectura no implica desresponsabilizar a los actores que deben garantizar el cumplimiento a cabalidad de los derechos de estos niños y niñas, sino invitar a pensar en la multiplicidad presente en sus construcciones identitarias y subjetivas y sus agentes relacionales como lo son la familia, la 
escuela y la comunidad. Esta invitación parte del reconocimiento de que dichas subjetividades e identidades se construyen en los significados compartidos.

La investigación social en el contexto del conflicto armado colombiano tiene el gran reto de aportar a la construcción de comprensiones acerca de la vida de los niños y niñas y de sus agentes relacionales significativos, desde miradas que amplíen el marco de comprensión y no lo reduzcan, y que involucren y visibilicen tanto los derechos no garantizados como los grandes aprendizajes, recursos y potencias presentes en ellos y en sus relaciones. La mirada del conflicto armado para la investigación debe atender a particularidades que permitan visibilizar las interacciones y el contexto a partir del aprecio por enfoques diferenciales y perspectivas complejas, como por ejemplo, las miradas por el género, la etnia y la generación entre otras, pues esto permite al investigador priorizar las significaciones desde lo particular y no desde lo universal, trascendiendo las explicaciones acostumbradas y los discursos con los cuales se ha comprendido durante mucho tiempo el conflicto armado.

La investigación social pertinente posibilita la comprensión de las motivaciones a la vinculación a las dinámicas del conflicto armado, o de la afectación que se presenta tras la vivencia directa o indirecta del conflicto, pero también posibilita la transformación y creación de realidades alternativas a la violencia, al partir de perspectivas que retoman los potenciales humanos y relacionales para la construcción de paz.

La pregunta por la forma en la que los niños y las niñas generan procesos de paz, democracia y reconciliación dentro de un contexto de conflicto armado hace necesario ver el contexto integral en el cual ellos y ellas social e históricamente han sido comprendidos a partir de múltiples dinámicas de interacción. Si bien este artículo retoma estudios en un periodo específico de 2002-2012, lo que se constitye en una limitación, al no incluir una revisión de los útimos cinco años y al contar con un número reducido de investigaciones incluidas en el ejercicio de estado del arte, permite generar cuestionamientos en torno a la influencia de los lenguajes en la comprensión de la niñez en conflicto armado, los cuales pueden limitarse a acciones en términos de protección y derechos o trascender hacia un lenguaje de las potencias que moviliza las posibilidades hacia otros caminos de construcción de paz.

En este sentido, también se cuestionan las narrativas hegemónicas del conflicto y el lugar que ocupa la comunidad científica, los medios de comunicación, las familias y otros agentes relacionales que desde el lenguaje influyen en la construcción de identidades y subjetividades de la niñez, abriendo o cerrando múltiples oportunidades de ser e incidir en el cambio. Aún quedan por develar muchas experiencias e historias de vida en las que los niños y las niñas demuestran el uso de sus capacidades de cambio.

\section{Referencias}

Alvarado, S., Luna, M. T., Ospina, H. F., Patiño, J. A., Quintero, M., \& Ospina, M. C. (2012). Las escuelas como territorios de paz, construcción social del niño y la niña como sujetos políticos en contextos de conflicto armado. Buenos Aires: Consejo Latinoamericano de Ciencias Sociales.

Arendt, H. (2005). La condición humana. Barcelona: Paidós.

Arendt, H. (2008). La promesa de la política. Barcelona: Paidós.

Berger, P., \& Luckmann, T. (1999). La construcción social de la realidad. Buenos Aires: Amorrortu.

Bronfrenbrenner, U. (1987). La ecología del desarrollo humano. Barcelona: Paidós.

Carli, S. (abril, 2003). El problema de la representación. Balances y dilemas. En actas del VII Seminario Internacional de Infancias y Adolescencias. Teorías y experiencias en el borde. Organizado por el Centro de Estudios Multidisciplinarios en Buenos Aires, Buenos Aires, Argentina.

Castellanos Obregón, J., \& Torres Silva, W. (2008). Una revisión de la producción 
académica sobre la violencia política en Colombia para indagar sobre el lugar de los jóvenes y las jóvenes. Revista Latinoamericana de Ciencias Sociales, Niñez y Juventud, 6(2), 523-563. Recuperado de http://redalyc.uaemex.mx/src/inicio/Art PdfRed.jsp?iCve $=77360204$

Coalición contra la vinculación de niños, niñas y jóvenes al conflicto armado en Colombia \& Comisión Colombiana de Juristas. (2009). Informe del Estado colombiano sobre el cumplimiento del protocolo facultativo relativo a la participación de los niños en conflictos armados (Informe alterno). Bogotá: Autores. Recuperado de http://ww w.coalico.org/archivo/IA10_EspIng.pdf

Defensoría del Pueblo. (2002). La niñez en el conflicto armado colombiano. Bogotá: UNICEF Colombia/Defensoría del Pueblo. Recuperado de http://www.unicef.org/colo mbia/conocimiento/boletn-8.htm

Defensoría del Pueblo. (2006). La niñez y sus derechos. Caracterización de las niñas, niños y adolescentes desvinculados de los grupos armados ilegales: inserción social y productiva desde un enfoque de derechos humanos. Bogotá: Unicef Colombia/ Defensoría del Pueblo. Recuperado de http://www.publicaciones.unicefcolomb ia.com/wpcontent/uploads/2006/03/Boleti n-defensoria.pdf

Gergen, K. (2007). Construccionismo social, aportes para el debate y la práctica. Bogotá: Ediciones Uniandes.

Hernández, A., \& Restrepo, M. (2011). Salud mental en niños en condición de desplazamiento en Colombia y su incidencia en políticas públicas. Bogotá: Universidad del Rosario.

Ila, P., Martínez, A., Arias, A., Núñez, P., \& Caicedo, M. (2009). Conflicto armado en la primera infancia en tres territorios colombianos: Putumayo, Magdalena Medio y Arauca. En A. Mejía (Ed.), Colombia: huellas del conflicto en la primera infancia (pp. 147-156). Bogotá: Revista Número.

Montoya, A. M. (2008). Niños y jóvenes en la guerra en Colombia. Aproximación a su reclutamiento y vinculación. Opinión Jurídica, 7(13), 37-51. Recuperado de http://www.udem.edu.co/NR/rdonlyres/ F1740B7A-0538-474D-9870-13A0729E52 05/10922/articulo2ninosyjovenes.pdf

Moreno, F., Carmona, J., \& Tobón, F. (2010). ¿Por qué se vinculan las niñas a los grupos guerrilleros y paramilitares en Colombia? Revista Latinoamericana de Psicología, 42(3), 453-457. Recuperado de http://ceanj.cinde.org.co/programa/Arc hivos/publicaciones/p1/_1_AN_154.pdf

Niño, N. (2012). Los derechos están torcidos. Una aproximación desde el enfoque de género y generacional a la vivencia de los derechos de las niñas en zonas de conflicto (Cúcuta y Tibú). Bogotá: Fundación Creciendo Unidos. Recuperado de http://www.natsper.org/upload/Los\%20 derechos\%20estan\%20torcidos.pdf

Observatorio de Territorios Étnicos. (2010). Una mirada desde la escuela al conflicto armado en San Onofre (Documento de trabajo). Bogotá: Autor. Recuperado de http://www.etnoterritorios.org/index.sht $\mathrm{ml}$ ?apc $=\mathrm{h} 1 \mathrm{e} 1 \ldots \mathrm{-} \& \mathrm{x}=662$

Organización de las Naciones Unidas. (2010). Los niños y los conflictos armados. Informe del Secretario General del Consejo de Seguridad (A-65-268S/2011/250). Ginebra: Autor. Recuperado de http://daccess-dds-ny.un.org/doc/UND OC/GEN/N11/275/36/PDF/N1127536.pdf ?OpenElement

Romero, T., \& Castañeda, E. (2009). El conflicto armado colombiano y la primera infancia. En A. Mejía (Ed.), Colombia: huellas del conflicto en la primera infancia (pp. 31-53). Bogotá: Revista Número.

Torrado, M., Camargo, M., Pineda, N., \& Bejarano, D. (2009). Estado del arte sobre primera infancia en el conflicto. En A. Mejía (Ed.), Colombia: huellas del conflicto en la primera infancia (pp. 31-53). Bogotá: Revista Número.

WATCHLIST on Children and Armed Conflict. (2004). Colombia: la guerra en los niños y las niñas (Informe). Nueva York: Autor. 
María Alejandra Fajardo Mayo, Mónica Piedad Ramírez Lozano, María Isabel Valencia Suescún, et al.

Recuperado de http://www.watchlist.org/re ports/pdf/colombia.report.es.pdf

\section{Notas}

* Artículo de revisión. Co-financiado por Colciencias y el Consorcio Niños, Niñas y Jóvenes Constructores de Paz: Democracia, paz y reconciliación; la Fundación CINDE;

la Universidad de Manizales y la Universidad

Pedagógica Nacional. 